\title{
Study of Torus Structure in Low-luminosity Active Galactic Nuclei with Suzaku
}

\section{Taiki Kawamuro*i}

Department of Astronomy, Kyoto University

E-mail: kawamuroekusastro.kyoto-u.ac.jp

\section{Yoshihiro Ueda}

Department of Astronomy, Kyoto University

E-mail: uedadkusastro.kyoto-u.ac.jp

\section{Fumie Tazaki}

National Astronomical Observatory of Japan

E-mail: fumie.tazakienao.ac.jp

\section{Yuichi Terashima}

Department of Physics, Ehime University

E-mail: terasimadastro.phys.sci.ehime-u.ac.jp

\section{Richard F. Mushotzky}

Department of Astronomy, University of Maryland

E-mail: richardeastro.umd.edu

\begin{abstract}
We report the results from a systematic study of the broad band X-ray spectra of low-luminosity active galactic nuclei (LLAGN) obtained with Suzaku and Swift/BAT. Our targets consist of NGC 1566, NGC 3718, NGC 4138, NGC 4941, and NGC 5273, all of which have the hard X-ray luminosities below $10^{42} \mathrm{erg} \mathrm{s}^{-1}$ in the $14-195 \mathrm{keV}$ band. Most of the spectra of the nucleus are well reproduced by a sum of partially or fully covered transmitted emission and its reflection from the accretion disk, reprocessed emission from the torus accompanied by a strong narrow iron-K $\alpha$ line, and a scattered component (for type-2 AGN). The equivalent width of the narrow iron-K $\alpha$ line enables us to constrain the covering fraction of the torus by comparing with a model prediction. We find that they generally agree with the obscured AGN fraction obtained from hard X-ray at similar luminosities. Our results support the implication that the averaged covering fraction of AGN tori is peaked at $L \sim 10^{42-43} \mathrm{erg} \mathrm{s}^{-1}$ but decreases toward lower luminosities.
\end{abstract}

10th INTEGRAL Workshop: "A Synergistic View of the High Energy Sky" - Integral2014,

15-19 September 2014

Annapolis, MD, USA

\footnotetext{
*Speaker.

$\dagger$ This work was partly supported by the Grant-in-Aid for JSPS Fellows for young researchers (T.K.)
} 


\section{INTRODUCTION}

Luminosity is a key parameter of an active galactic nucleus (AGN) that determines the physical conditions of the central region. Thus, to understand the AGN phenomena, it is important to observe them over a wide luminosity range and establish their properties as a function of luminosity. In spite of the fact that low-luminosity AGN (LLAGN) are common in the local universe (e.g., Ho 2008), the detailed study of broad band X-ray spectra of LLAGN has been limited due to their faintness (for previous works below $10 \mathrm{keV}$, see e.g., Terashima et al. 2002).

There is an anti-correlation between the obscured (type-2) AGN fraction and luminosity (see e.g., Hasinger 2008, Ueda et al. 2014). This indicates that the covering fraction of obscuring material around a SMBH becomes larger in less luminous AGN. Recent studies using hard X-ray ( $>15 \mathrm{keV}$; Beckmann et al. 2009, Burlon et al. 2011) selected samples suggest that this relation becomes more complex when much lower luminosity AGN are included, although the sample size is small. The obscured fraction has a peak of $\approx 0.6-0.8$ around the $15-55 \mathrm{keV}$ luminosity (hereafter $L_{15-55}$ ) of $\sim 10^{42-43} \mathrm{erg} \mathrm{s}^{-1}$ below which it starts to decrease toward lower luminosity. The physics that determines the torus structure of LLAGN with $L_{15-55}<10^{42} \mathrm{erg} \mathrm{s}^{-1}$ may be different from that of typical Seyfert galaxies with $L_{15-55} \sim 10^{43} \mathrm{erg} \mathrm{s}^{-1}$.

Broad band X-ray spectra provide us with valuable information of the primary X-ray continuum and its reprocessed emission from the surrounding matter around the SMBH e.g., dust torus and accretion disk. In this paper, we present the results of a systematic spectral analysis of five nearby LLAGN, NGC 1566, NGC 3718, NGC 4138, NGC 4941, and NGC 5273, using Suzaku and Swift/BAT data. The redshift and optical classification of our targets are represented in Table $\mathrm{W}$. These AGN have $L_{15-55} \sim 10^{41-42} \mathrm{erg} \mathrm{s}^{-1}$ and were selected from the Swift/BAT catalog (Baumgartner et al. 2013), which ensures sufficiently bright hard X-ray fluxes above $10 \mathrm{keV}$ to obtain good signal to noise with the Suzaku PIN. The primary aim of our study is to constrain their torus geometry by precise observations of the iron-K $\alpha$ emission line. The results for NGC 1566 and NGC 4941 have been reported in Kawamuro et al. (2013).

We adopt distances of 16.5, 14.2, 12.7, 19.7, and 15.0 Mpc for NGC 1566, NGC 3718, NGC 4138, NGC 4941 and NGC 5273, respectively, in calculating the luminosities. We apply the Galactic absorption estimated from the H I map (Kalberla et al. 2005) for each AGN. The solar abundances by Anders \& Grevesse (1989) are assumed. The errors attached to spectral parameters are given at $90 \%$ confidence limits for a single parameter of interest.

\section{ANALYSIS}

\subsection{Broadband Spectral Analysis}

Spectral analysis is performed by utilizing the data of FI-XISs (XIS0 + XIS3), BI-XIS (XIS1), and HXD/PIN onboard Suzaku, and those of BAT onboard Swift averaged for 70 months, in the energy band of 1-12 keV, 0.5-8 keV, 16-60 keV, and 14-195 keV, respectively. Figure $\square$ plots the Suzaku unfolded spectra of NGC 1566 (type-1 AGN) and of NGC 4941 (type-2 AGN) as examples.

As a baseline model, we adopt the same model used by Tazaki et al. (2013), which is composed of four components for the nucleus emission: (1) the primary transmitted component from the nucleus, (2) the reflection component from the accretion disk, (3) that from the torus, and 
Table 1: Target Information

\begin{tabular}{cccc}
\hline \hline Target Name & Swift Name & Redshift & Class \\
\hline NGC 1566 & SWIFT J0420.0-5457 & 0.00502 & Seyfert 1.5 \\
NGC 3718 & SWIFT J1132.7+5301 & 0.00331 & LINER 1.9 \\
NGC 4138 & SWIFT J1209.4+4340 & 0.00296 & Seyfert 1.9 \\
NGC 4941 & SWIFT J1304.3-0532 & 0.0037 & Seyfert 2 \\
NGC 5273 & SWIFT J1341.9+3537 & 0.0035 & Seyfert 1.5 $1.5^{1} 1.9^{1}$ \\
\hline
\end{tabular}

${ }^{1}$ Véron-Cetty, M.-P. and Véron, P. 2006.

${ }^{2}$ Ho et al. 1997.
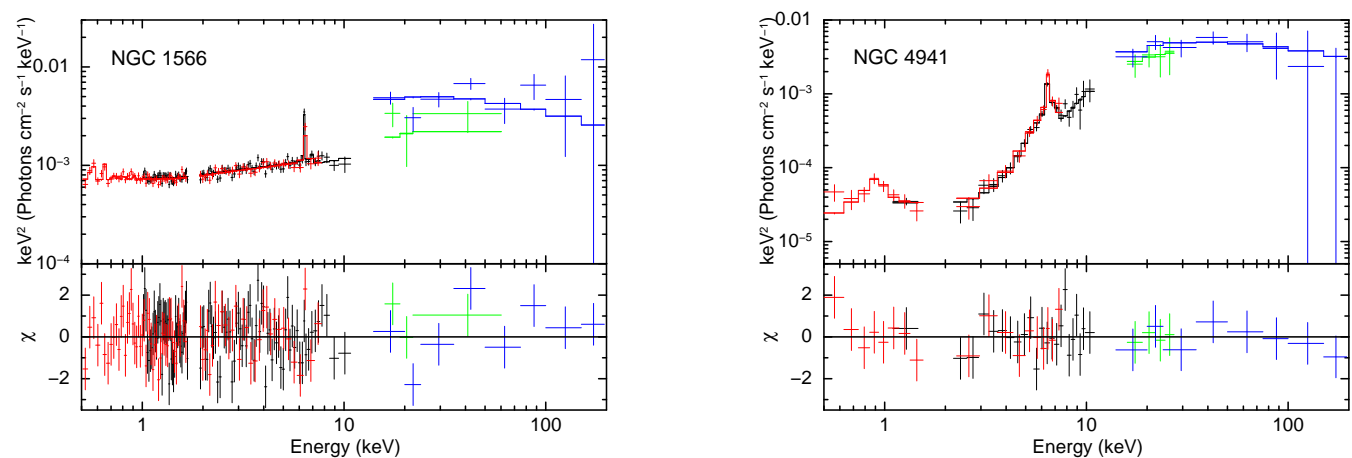

Figure 1: (Left Figure): Suzaku and Swift/BAT spectra of NGC 1566 (crosses). Black, red, green, and blue represent the FI-XISs, BI-XIS, HXD/PIN, and Swift/BAT spectra, respectively. The Suzaku spectra are not folded with the energy response. The fitting residuals in units of $\chi$ are shown in the lower panel. (Right Figure): The same plots for NGC 4941.

(4) the scattered component from surrounding gas in the case of absorbed AGN. In four targets (NGC 1566, NGC 4138, and NGC 4941), we also consider emission from an optically-thin thermal plasma in the host galaxy, which is often observed from LLAGN as "soft excess" below $\sim 1$ $\mathrm{keV}$ (Terashima et al. 2002). To model it, we use the apec (Smith et al. 2001) model in XSPEC. We approximate the shape of the primary continuum with an exponential-cutoff power-law model, $E^{-\Gamma} \exp \left(-E / E_{\text {cut }}\right)$. Since it is difficult to constrain $E_{\text {cut }}$ from our data, we fix it at $360 \mathrm{keV}$ for consistency with the torus model by Ikeda et al. (2009).

Due to variability of X-ray emission, it is expected that the flux of the direct component changes between the short ( $\sim 2$ days) Suzaku observation and the long (70 months) Swift/BAT observation. To take it into account, a normalization factor of Suzaku (FI-XISs) relative to the Swift/BAT, $N_{\mathrm{XIS}}$, is introduced as a free parameter, by assuming that the continuum shape (i.e., $\Gamma$ and $\left.E_{\text {cut }}\right)$ is constant. The same factor is also applied to the reflection component from the accretion disk, while it is not applied to that from the torus (except for NGC 5273), the scattered component, and the thin thermal emission, assuming that their fluxes do not change over years because of their large spatial scales. In NGC 5273, we find that the flux of the narrow iron-K $\alpha$ line in the Suzaku observations performed in 2013 July significantly decreases compared with that observed with XMM-Newton in 2002 June. This can be explained if we assume that the reflection 


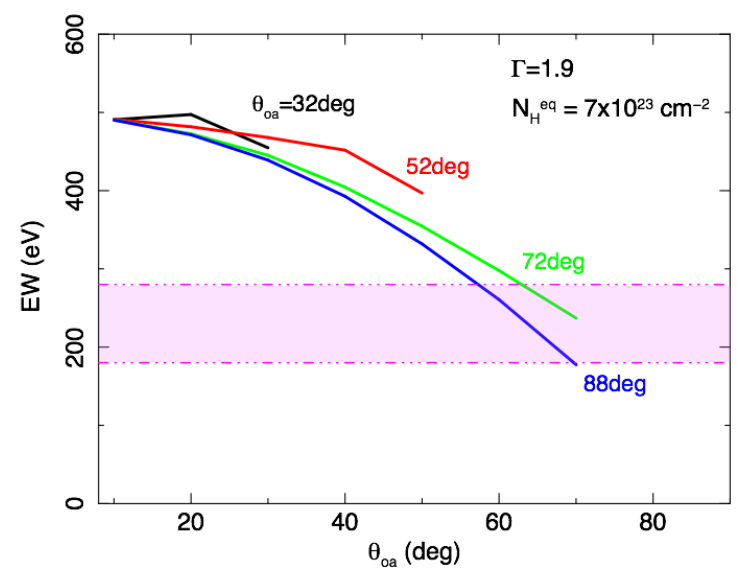

Figure 2: Predicted equivalent widths of iron-K $\alpha$ line for type-2 AGN plotted as a function of torus halfopening angle, based on the torus model by Ikeda et al. (2009). An equatorial column density of $N_{\mathrm{H}}^{\mathrm{eq}}=$ $7 \times 10^{23} \mathrm{~cm}^{-2}$ and a photon index of 1.9 are assumed. Several different inclinations are assumed as labeled in the figure. The black, red, green, and blue represent the results at the inclination angle $\theta_{\text {inc }}=32^{\circ}, 52^{\circ}, 72^{\circ}$ and $88^{\circ}$, respectively. The $90 \%$ confidence upper and lower limits for the corrected iron-K $\alpha$ line equivalent width (see text) of NGC 4941 are shown by the two horizontal lines with the shaded region (magenta).

component from the dust torus follows the variability of the direct component within $\sim$ one year.

Two reflection components from the accretion disk and dust torus should be taken into consideration. To model them, we utilize the pexmon model (Nandra et al. 2007), which consists of the reflected continuum based on the pexrav model (Magdziarz \& Zdziarski 1995) and fluorescence lines (including iron-K $\alpha$ ) self-consistently calculated with the continuum. The only free parameter is the reflection strength $R \equiv \Omega / 2 \pi$, where $\Omega$ is the solid angle of the reflector covering the X-ray source. Because the reflection component from the accretion disk should be subject to relativistic effects, we convolve it with the rdblur model. To avoid the coupling between the two reflection components from the torus and accretion-disk in the spectral fit, we fix the reflection strength from the accretion disk $R_{\text {disk }}$ by assuming a simple geometry of the disk and corona (for details, see Kawamuro et al. 2013).

\subsection{Analysis of Iron-K $\alpha$ Line in Narrow Band Spectra}

To verify the broad-band fit results, we also perform spectral analysis in the $3-9 \mathrm{keV}$ band, focusing on the iron-K $\alpha$ line features. The best-fit continuum model obtained by the broad-band fit is adopted by replacing the pexmon components with the pexrav model, which does not contain fluorescence lines. Instead, we add a diskline component (Fabian et al. 1989) as an iron-K $\alpha$ emission line from the accretion disk assuming an innermost radius of $100 r_{\mathrm{g}}$ (where $r_{\mathrm{g}}$ is the gravitational radius) and a power-law emissivity index of -3 , and zgauss as that from the torus. The line energies for both components are fixed at $6.4 \mathrm{keV}$ in the rest frame. Only the normalizations of diskline, zgauss, and zpowerlw are set free.

The observed equivalent width $\mathrm{EW}_{\mathrm{Gauss}}^{\mathrm{obs}}$ of the narrow iron-K $\alpha$ line (zgauss) from the torus with respect to the total continuum is subject to variability of the transmitted component. From the broad band spectral analysis, we know that the fluxes during the Suzaku observations were much fainter than those of Swift/BAT in some targets. This leads to an overestimate of the true 
(i.e., time averaged) equivalent widths of the iron- $\mathrm{K} \alpha$ line. On the basis of our assumption that the long-term Swift/BAT data give the averaged continuum flux, we calculate the true continuum level at $6.4 \mathrm{keV}$ by increasing the transmitted component by a factor of $1 / N_{\mathrm{XIS}}$, and then derive "corrected" equivalent width $\mathrm{EW}_{\mathrm{Gauss}}^{\text {cor }}$. In the case of NGC 4941, the observed equivalent width and the corrected one are $380 \pm 80 \mathrm{eV}$ and $230 \pm 50 \mathrm{eV}$, respectively.

\section{DISCUSSION}

The equivalent width of the narrow iron-K $\alpha$ line from an AGN carries useful information to constrain the torus structure, such as the opening angle. For this purpose, we utilize the MonteCarlo based numerical model by Ikeda et al. (2009), which calculates the reflected continuum with fluorescence lines from an AGN surrounded by a torus. In this model, the geometry of the torus is close to be a spherical shape defined by three parameters (for details see Figure 2 of Ikeda et al. (2009)): hydrogen column density at the equatorial plane $N_{\mathrm{H}}^{\mathrm{eq}}$, half opening angle $\theta_{\mathrm{oa}}$, and inclination $\theta_{\text {inc. Hence, }} \theta_{\text {inc }}<\theta_{\text {oa }}$ for type- 1 AGN and $\theta_{\text {inc }}>\theta_{\text {oa }}$ for type- 2 AGN. As done in Tazaki et al. (2013), we can plot the predicted equivalent width of the iron-K $\alpha$ line as a function of half opening angle with several different inclinations. Figure $\square$ gives an example for a type-2 AGN; here we adopt $N_{\mathrm{H}}^{\mathrm{eq}}=7 \times 10^{23} \mathrm{~cm}^{-2}$ and $\Gamma=1.9$, the best fit line-of-sight column density and photon index of NGC 4941. The dashed horizontal lines (magenta) in Figure $\square$ represent the error region of the corrected equivalent width $\mathrm{EW}_{\mathrm{G}}^{\mathrm{cou}}$ for $\mathrm{NGC}$ 4941. Then, the half opening angle is estimated to be $\theta_{\mathrm{oa}} \simeq 60^{\circ}-70^{\circ}$ from the iron-K $\alpha$ equivalent width, which is consistent with $R_{\text {torus }}=0.64_{-0.27}^{+0.69}$.

According to the unified scheme, the torus covering fraction determines the fraction of obscured AGN. Thus, it is interesting to compare our results with the type-2 fraction derived from unbiased AGN surveys. On the basis of the Swift/BAT survey performed in the $15-55 \mathrm{keV}$ band, Burlon et al. (2011) suggest that the fraction has a peak of $\approx 0.6-0.8$ around $L_{15-55}=10^{42-43} \mathrm{erg}$ $\mathrm{s}^{-1}$ and decreases toward both higher and lower luminosity ranges. At $L_{15-55}=10^{41-42} \mathrm{erg} \mathrm{s}^{-1}$, the fraction is estimated to be $0.1-0.6$ (see e.g., their Figure 16), although the error is still quite large due to a limited number of LLAGN detected in the hard X-ray survey. Based on the best-fit model, the $15-55 \mathrm{keV}$ luminosity of NGC 4941 is $4.5 \times 10^{41} \mathrm{erg} \mathrm{s}^{-1}$. Our result on the torus halfopening angle, $\theta_{\mathrm{oa}} \simeq 60^{\circ}-70^{\circ}$, can be converted into an obscured fraction of $0.34-0.50$, which is fully consistent with the above survey results at a similar luminosity range.

Another good indicator of the torus covering fraction is the ratio of the luminosity of the iron$\mathrm{K} \alpha$ line to that of the continuum in the $10-50 \mathrm{keV}$ band, where the effect of absorption can be mostly neglected. Using Suzaku spectra of nearby Seyfert galaxies, Ricci et al. (2014) study the luminosity dependence of this parameter, as shown in Figure [1]. In the figure, we overplot the results obtained from our LLAGN. For these targets except NGC 5273, we define the continuum luminosity as the absorption-corrected one observed with Swift/BAT to take into account time variability of the continuum flux. Figure B] shows the trend that the iron-K $\alpha$ line to continuum luminosity ratio increases with decreasing luminosity down to $\sim 10^{42-43} \mathrm{erg} \mathrm{s}^{-1}$ but then decreases toward the lower luminosity range. This suggests that the torus covering fraction is peaked at $L_{10-50} \sim 10^{42-43}$ $\mathrm{erg} \mathrm{s}^{-1}$, which is consistent with the above argument based on the obscured AGN fraction. To con- 
firm our results and to understand the origin, further studies of a larger sample of LLAGN covering a wide range of luminosity and Eddington ratio would be useful.

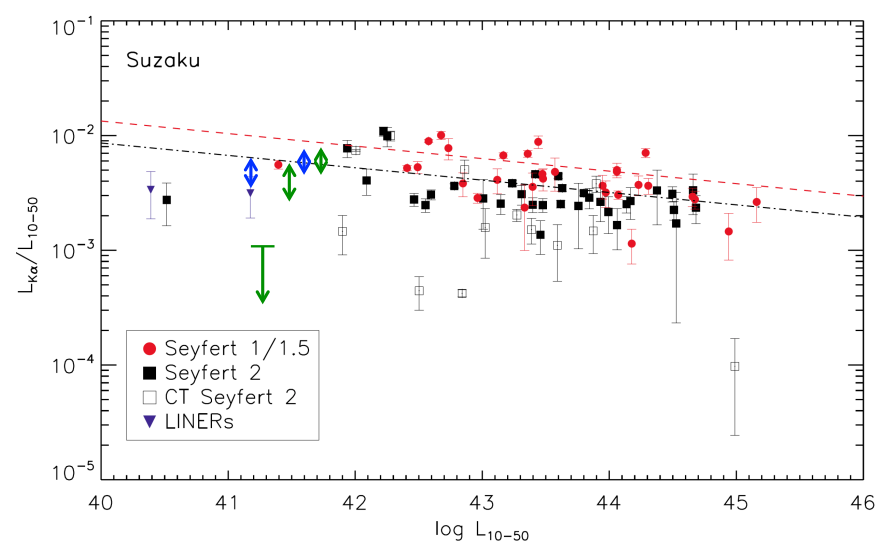

Figure 3: The ratio of the luminosity of the iron- $\mathrm{K} \alpha$ line to that of the $10-50 \mathrm{keV}$ continuum plotted against the 10-50 keV luminosity for nearby AGN, updated from that in Ricci et al. (2014). The filled circles (red) correspond to Seyfert 1/1.5, the filled squares (black) Seyfert 2, the open squares Compton-thick Seyfert 2, and the filled triangles Low-ionization nuclear emission-line region. The blue and green arrows represent our results of type-1 LLAGN and type-2 LLAGN, respectively.

\section{References}

[1] Anders, E., \& Grevesse, N. 1989, GeCoA, 53, 197

[2] Baumgartner, W. H., Tueller, J., Markwardt, C. B., et al. 2013, ApJS, 207, 19

[3] Beckmann, V. et al. 2009, A\&A, 505, 417

[4] Burlon, D., Ajello, M., Greiner, J., et al. 2011, ApJ, 728, 58

[5] Fabian, A. C., Rees, M. J., Stella, L., \& White, N. E. 1989, MNRAS, 238, 729

[6] George, I. M., \& Fabian, A. C. 1991, MNRAS, 249, 352

[7] Hasinger, G., 2008, A\&A, 490, 905

[8] Ho, L. C., Filippenko, A. V., Sargent, W. L. W., \& Peng, C. Y. 1997, ApJS, 112, 391

[9] Ho, L. C. 2008, ARA\&A, 46, 475

[10] Ikeda, S., Awaki, H., \& Terashima, Y. 2009, ApJ, 692, 608

[11] Kalberla, P. M. W., Burton, W. B., Hartmann, D., et al. 2005, A\&A, 440, 775

[12] Kawamuro, T., Ueda, Y., Tazaki, F., \& Terashima, Y. 2013, ApJ, 770, 157

[13] Magdziarz, P., \& Zdziarski, A. A. 1995, MNRAS, 273, 837

[14] Nandra, K., O’Neill, P. M., George, I. M., \& Reeves, J. N. 2007, MNRAS, 382, 194

[15] Ricci, C., Ueda, Y., Paltani, S., et al. 2014, MNRAS, 441, 3622

[16] Smith, R. K., Brickhouse, N. S., Liedahl, D. A., \& Raymond, J. C. 2001, ApJL, 556, L91

[17] Tazaki, F., Ueda, Y., Terashima, Y., \& Mushotzky, R. F., Tombesi, F. 2013, submitted to ApJ 
[18] Terashima, Y., Iyomoto, N., Ho, L. C., \& Ptak, A. F. 2002, ApJS, 139, 1

[19] Ueda, Y., Akiyama, M., Hasinger, G., Miyaji, T., \& Watson, M. G. 2014, ApJ, 786, 104

[20] Véron-Cetty, M.-P., \& Véron, P. 2006, A\&A, 455, 773 